\title{
COPULA GAUSSIAN GRAPHICAL MODELS WITH HIDDEN VARIABLES
}

\author{
Hang Yu, Justin Dauwels, and Xueou Wang \\ School of Electrical and Electronics Engineering, School of Physical and Mathematical Sciences \\ Nanyang Technological University, Singapore, 639798
}

\begin{abstract}
Gaussian hidden variable graphical models are powerful tools to describe high-dimensional data; they capture dependencies between observed (Gaussian) variables by introducing a suitable number of hidden variables. However, such models are only applicable to Gaussian data. Moreover, they are sensitive to the choice of certain regularization parameters. In this paper, (1) copula Gaussian hidden variable graphical models are introduced, which extend Gaussian hidden variable graphical models to non-Gaussian data; (2) the sparsity pattern of the hidden variable graphical model is learned via stability selection, which leads to more stable results than crossvalidation and other methods to select the regularization parameters. The proposed methods are validated on synthetic and real data.
\end{abstract}

Index Terms - Gaussian copula, hidden variable graphical model, stability selection, bioinformatics

\section{INTRODUCTION}

Sparse graphical models (see, e.g., [1]) provide an effective way to capture statistical structure in high-dimensional data, such as gene expression data, multi-electrode brain recordings, and stock market data. A sparse graph displays the most significant interactions between variables (e.g., genes, brain areas, stocks), and may help to interpret the data.

In practice, it is quite common that data is unavailable for some relevant variables. For instance, one typically measures the expression of a limited subset of genes, chosen among the large number of genes of an organism; the observed genes may be strongly affected by genes that have not been measured. The latter may then be treated as hidden variables in a statistical model, providing a simple explanation for the statistical relations between the observed genes.

Therefore, sparse graphical models with hidden variables are quite powerful models for a large variety of real-life datasets. When the observed variables $Z_{o}$ and hidden variables $Z_{h}$ are jointly Gaussian distributed, the structure of the graphical model can be defined by the precision matrix (inverse covariance matrix) of the observed and hidden variables. Recently, Chandraskaran et al. [2] decomposed the (marginal) precision matrix of $Z_{o}$ into a sparse matrix $K_{o}$ (conditional precision matrix) and a low-rank matrix $L$, which describes the coupling between the observed and hidden variables. The conditional graphical model $K_{o}$ and the number of hidden variables (rank of $L$ ) are inferred by solving a convex regularized maximum-likelihood problem. The conditional precision matrix $K_{o}$ is represented as a graph, where nodes $i$ and $j$ are connected by an edge iff the corresponding element $(i, j)$ in $K_{o}$ is non-zero. That graph visualizes the dependence among the observed variables, conditioned on the hidden variables.

Obviously, Gaussian hidden variable graphical models (GHVGM) are limited to Gaussian data. In this paper, we extend GHVGM to non-Gaussian data by means of Gaussian copulas [3], referred to as copula Gaussian hidden variable graphical model (CGHVGM).

Another issue with the GHVGM is the selection of the regularization/penalty parameters, which determine the resulting sparsity pattern of $K_{o}$ and rank of $L$. Standard approaches for regularization selection, including cross validation (cv), Akaike's information criterion (AIC), and Bayesian information criterion (BIC), are known to overfit the data, and they typically result in graphs that are too dense [4].

In this paper, we circumvent the delicate issue of regularization selection by first learning the graph structure and next inferring the parameters. Specifically, stability selection [5] is used to learn the structure (sparsity pattern) of $K_{o}$. We further specify $K_{o}$ and $L$ by solving a convex problem subject to the structure constraints. The estimated number of hidden variables equals to the rank of $L$.

We apply our model (CGHVGM) to non-Gaussian synthetic and real data (cell signaling data). The CGHVGM is able to recover the number of hidden variables and the conditional graph $K_{o}$, in contrast to other related models. Interestingly, the GHVGM dramatically failed for both datasets, presumably because it is not intended for non-Gaussian data.

This paper is organized as follows. In Section 2, we first review the copula Gaussian graphical model and the Gaussian hidden variable graphical model, and next we present the proposed copula Gaussian hidden variable graphical model. In Section 3, we explain how we learn the structure and parameters of the proposed model. In Section 4, we assess the proposed model and benchmark it with other models, by means of synthetic and real data. In Section 5, we offer concluding remarks. 


\section{GRAPHICAL MODELS}

In the following, we briefly describe the copula Gaussian graphical model, the Gaussian hidden variable graphical model, and the proposed copula Gaussian hidden variable graphical model.

\subsection{Copula Gaussian Graphical Model}

We denote the observed non-Gaussian variables and hidden Gaussian variables as $Y_{1}, \ldots, Y_{P}$ and $Z_{1}, \ldots, Z_{P}$ respectively. A Gaussian copula graphical model is defined as [3]:

$$
\begin{aligned}
Z & \sim \mathcal{N}\left(0, K^{-1}\right) \\
Y_{k} & =F_{k}^{-1}\left(\Phi\left(Z_{k}\right)\right),
\end{aligned}
$$

where $K$ is the precision matrix whose inverse (the covariance matrix) has normalized diagonal, $\Phi$ is the CDF (cumulative distribution function) of the standard Gaussian distribution, and $F_{k}$ is the CDF of $Y_{k}$. The latter is often approximated by the empirical distributions $\hat{F}_{k}$. Note that $F_{k}^{-1}$ is the pseudo-inverse of $F_{k}$, which is defined as:

$$
F^{-1}(y)=\inf _{x \in \mathcal{X}}\{F(x) \geq y\} .
$$

\subsection{Gaussian Hidden Variable Graphical Model}

Suppose we have Gaussian distributed observed variables $Z_{o}$ and hidden variables $Z_{h}$. The joint precision matrix $K_{(o h)}$ associated with these variables is given by:

$$
K_{(o h)}=\left[\begin{array}{cc}
K_{o} & K_{o, h} \\
K_{h, o} & K_{h}
\end{array}\right] .
$$

According to Schur complement, the marginalized precision matrix $\tilde{K}_{o}$ of $Z_{o}$ can be written as:

$$
\tilde{K}_{o}=K_{o}-K_{o, h} K_{h}^{-1} K_{h, o}=K_{o}-L,
$$

with product matrix $L=K_{o, h} K_{h}^{-1} K_{h, o}$. Those two components have their own properties [2]: $K_{o}$ is the supposedly sparse conditional precision matrix of $Z_{o}$, conditioned on $Z_{h}$; the product matrix $L$ summarizes the effect of marginalization over the hidden variables. The rank of that matrix (equal to the number of hidden variables $Z_{h}$ ) is low, since the number of hidden variables is supposed to be small.

Given i.i.d. samples of $Z_{o}$, our objective is to estimate $K_{o}$ and $L$; we are especially interested in the rank of $L$, since it equals the number of hidden variables $Z_{h}$. Those matrices may be recovered by solving the convex relaxation [2]:

$$
\begin{aligned}
\left(\hat{K}_{o}, \hat{L}\right) & =\underset{K_{o}, L}{\operatorname{argmin}} \operatorname{trace}\left(\left(K_{o}-L\right) \Sigma_{o}\right)-\log \operatorname{det}\left(K_{o}-L\right) \\
& +\lambda\left(\gamma\left\|K_{o}\right\|_{1}+\operatorname{trace}(L)\right),
\end{aligned}
$$

where $\hat{K}_{o}$ and $\hat{L}$ are the estimates of $K_{o}$ and $L$ respectively, and $\Sigma_{o}$ is the empirical marginal covariance of $Z_{o}$. The convex problem (6) can be solved efficiently by the Newton-CG primal proximal point algorithm [6]. To recover the correct matrices $K_{o}$ and $L$, the parameters $\lambda$ and $\gamma$ need to be chosen appropriately, which is a critical issue that will be addressed in Section 3.

\subsection{Copula Gaussian Hidden Variable Graphical Model}

The observed (continuous) variables $Y$ are non-Gaussian, and each of them is associated with a Gaussian distributed hidden variable $Z_{o}$, as in the copula Gaussian model. However, besides the hidden variables $Z_{o}$, there exist several hidden variables $Z_{h}$ that are not associated with observed variables. In the graphical model, the nodes $Z_{h}$ are only connected to hidden variables; they are not connected to observed variables $Y$. In other words, the variables $Y$ and $Z_{o}$ constitute a Gaussian copula graphical model, while the variables $Z_{o}$ and $Z_{h}$ form a Gaussian hidden variable graphical model; together, the variables $\left(Y, Z_{o}, Z_{h}\right)$ form a copula Gaussian hidden variable graphical model with associated conditional precision matrix $K_{o}$ and product matrix $L$ (cf. (5)).

Given i.i.d. samples of the non-Gaussian variables $Y$, we wish to infer the conditional precision matrix $K_{o}$ of $Z_{o}$ (conditioned on $Z_{h}$ ), and the product matrix $L$.

As a first step, we transform the non-Gaussian observed variables $Y$ into Gaussian distributed hidden variables $Z_{o}$ (associated with the observed variables $Y$ ):

$$
Z_{o k}=\Phi^{-1}\left(\hat{F}_{k}\left(Y_{k}\right)\right)
$$

where $\Phi$ is the CDF of the standard Gaussian distribution and $\hat{F}_{k}$ is the empirical CDF of $Y_{k}$. As a result, we are dealing with Gaussian variables $Z_{o}$ which together with $Z_{h}$ constitute a GHVGM.

In the second step, we follow the procedure of (6) to infer the sparse conditional precision matrix $K_{o}$ of $Z_{o}$ and the lowrank product matrix $L$. Also here, of course, we need to pay special attention to the parameters $\lambda$ and $\gamma$, which will be the subject of Section 3.

\section{LEARNING AND INFERENCE}

A suitable choice of regularization parameters $\lambda$ and $\gamma$ in (6) can produce the graphical model with true sparsity pattern of $K_{o}$. However, standard procedures for selecting $\lambda$ and $\gamma$ are known to overfit the data and result in graphs that are too dense [4]. As an alternative, we employ a two-step procedure of structure learning and parameter learning. Stability selection [5] is used for structure learning, resulting in the sparsity pattern of $K_{o}$; constrained by this inferred sparsity pattern, we then infer the parameters by solving a problem similar to (6).

\subsection{Structure Learning}

We use the stability selection procedure [5] to infer the sparsity pattern of the conditional precision matrix $K_{o}$, from $N$ i.i.d. samples $S$ of $Y \in \mathbb{R}^{P}$ (or $Z_{o} \in \mathbb{R}^{P}$ ). 
First, $M$ subsets $S_{1}, S_{2}, \ldots, S_{M}$ are randomly drawn without replacement from the dataset, each of size $\lfloor N / 2\rfloor$.

Second, we select a range of $\lambda$ and $\gamma$ (cf. (6)). Now focus on one pair of parameters $(\lambda, \gamma)$ in that range. For each subset $S_{m}$ (for $m=1, \ldots, M$ ), we estimate one precision matrix $K_{o}$ using (6), resulting in $M$ precision matrices $K_{1}, \ldots$, $K_{M}$. For each element $(i, j)$ in the matrix $K_{m}$, the number of times it is non-zero $\left(K_{m}(i, j) \neq 0\right)$ among the $M$ matrices is counted and divided by $M$; as a result, we obtain the probability (stability) that this edge exists in the graphical model associated with $(\lambda, \gamma)$. By varying $\lambda$ and $\gamma$ through the chosen range, we can draw a surface of the stability for each edge.

At last, we include edge $(i, j)$ in the graphical model associated with $K_{o}$, if the probability of that edge, for at least one pair $(\lambda, \gamma)$ in the selected range, is larger than threshold $\pi_{t h r}[5]:$

$$
\pi_{t h r}=\frac{\bar{p}^{2}}{P(P-1) E}+0.5 .
$$

The parameter $\bar{p}$ is the average number of edges in the graphs associated with each pair $(\lambda, \gamma)$ in the selected range, inferred from the entire dataset $S$ through (6). $E$ is the expected number of falsely selected edges.

We also applied a randomized approach suggested in [5]. When inferring the matrices $K_{m}$, we divide the parameter pair $(\lambda, \gamma)$ by a random pair $\left(\alpha_{1}, \alpha_{2}\right)$ (different for each subset), where $\alpha_{1}$ and $\alpha_{2}$ are uniformly distributed on $[0.2,1]$. In the following, we will only report results for the randomized approach, since it yields the best results.

\subsection{Parameter Learning}

The structure (sparsity pattern) of $K_{o}$ has now been inferred, and that helps us to estimate $K_{o}$ and $L$; specifically, we solve a problem similar to (6), where the $l_{1}$ term is removed, and the sparsity pattern is encoded by a large penalty $\sigma$ on the absolute value of zero elements in $K_{o}$ :

$$
\begin{aligned}
\left(\hat{K}_{o}, \hat{L}\right) & =\underset{K_{o}, L}{\operatorname{argmin}} \operatorname{trace}\left(\left(K_{o}-L\right) \Sigma_{o}\right)-\log \operatorname{det}\left(K_{o}-L\right) \\
& +\lambda \operatorname{trace}(L)+\sigma \sum_{K_{o}(i, j)=0}\left|K_{o}(i, j)\right| .
\end{aligned}
$$

The parameter $\lambda$ is selected as the mean of the $\lambda \mathrm{s}$ in the chosen range (the second step of stability selection) that generate the same structure as $K_{o}$ estimated by stability selection. The number of hidden variables can be estimated easily by computing the rank of $L$.

\section{NUMERICAL RESULTS}

We test our proposed graphical model and existing graphical models on a synthetic and real data set.

\subsection{Synthetic Data}

We generate non-Gaussian synthetic data using the following method:
1. Generate a random precision matrix by using the method of [7], which mimics characteristics of realworld biological networks. More specifically, we first uniformly sample $x_{1}, \ldots, x_{n}$ from a unit square. The precision matrix is initialized as a unit matrix. Next, we set the element $K(i, j)=K(j, i)$ of precision matrix equal to $\rho=0.245$ with probability $(\sqrt{2 \pi})^{-1} \exp \left(-4\left\|x_{i}-x_{j}\right\|^{2}\right)$, and equal to zero otherwise.

2. Add a few variables and connect each of them to at least $80 \%$ of other variables (corresponding elements in precision matrix are non-zero).

3. Generate a Gaussian dataset corresponding to the above precision matrix and discard all the samples of variables added in Step 2 (hidden variables).

4. Apply different types of copula to each variable (including beta, exponential, chi-square copula), transforming the Gaussian variables to (continuous) nonGaussian variables.

We apply our proposed graphical model to that data, in particular, copula Gaussian hidden variable graphical model selection with stability selection (CGHVGM with ss). We also consider 6 other approaches including glasso [1], copula glasso [7], Gaussian hidden variable graphical model inferred by cross validation (GHVGM with cv), Gaussian hidden variable graphical model with stability selection (GHVGM with ss), and copula Gaussian hidden variable graphical model selection with cross validation (CGHVGM with cv). We evaluate those methods through various criteria including precision, recall, $F_{1}$-score, and number of parameters (Prm No.). Precision is defined as the proportion of correctly estimated edges to all the edges in the estimated graph; recall is defined as the proportion of successfully estimated edges to all the edges in the true graph; $F_{1}$-score is defined as 2 precision.recall/(precision+recall), which is a weighted average of the precision and recall.

The results for a 20-dimensional dataset are summarized in Fig. 1 and Table 1.

Table 1. Quantitative comparison of different methods

\begin{tabular}{ccccc}
\hline \hline \multirow{2}{*}{ Methods } & \multicolumn{4}{c}{ Criteria } \\
\cline { 2 - 5 } & Precision & Recall & $F_{1}$-score & Prm No. \\
\hline glasso & 0.1195 & 0.9048 & 0.2111 & 179 \\
copula glasso & 0.1105 & 1.0000 & 0.1990 & 210 \\
GHVGM with cv & 0.1324 & 0.4286 & 0.2023 & 488 \\
GHVGM with ss & 0.0000 & 0.0000 & 0.0000 & 0 \\
CGHVGM with cv & 0.2877 & 1.0000 & 0.4468 & 173 \\
CGHVGM with ss & 0.8750 & 1.0000 & 0.9333 & 64 \\
\hline glasso(m) & 1.0000 & 0.6737 & 0.8050 & 179 \\
copula glasso(m) & 1.0000 & 1.0000 & 1.0000 & 210 \\
\hline \hline
\end{tabular}

The results show that CGHVGM with ss achieves the best performance with the least number of parameters. The CGHVGM approach with cv generates a dense graph, whereas GHVGM with ss produces graph without edges. 


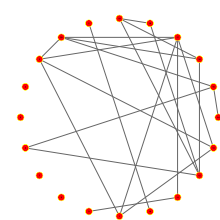

(a) True graph

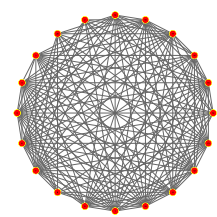

(b) Glasso

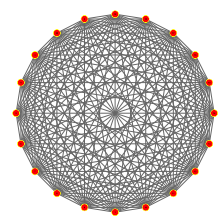

(c) Copula glasso

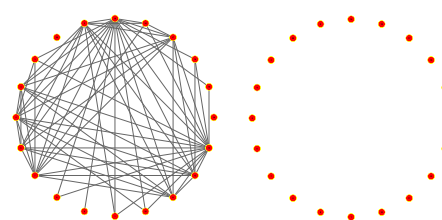

(d) GHVGM(cv)

(e) GHVGM(ss)

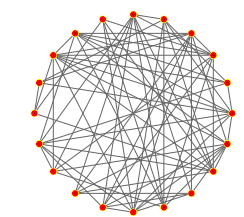

(f) CGHVGM(cv)

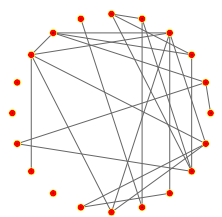

(g) CGHVGM(ss)

Fig. 1. Results of different methods on the 20-dimensional synthetic dataset

The rank of the inferred $L$ in CGHVGM with ss equals to the number of hidden variables. However, the rank estimated using CGHVGM with $\mathrm{cv}$ is four times larger than the true value, while GHVGM with cv yields a full-rank matrix $L$.

The glasso and copula glasso methods infer the marginal precision matrix $\tilde{K}_{o}$ in (5), instead of the conditional precision matrix $K_{o}$. The results for that inference problem are listed under glasso(m) and copula glasso(m) in Table 1, where " $\mathrm{m}$ " refers to marginal. The performance of copula glasso is the best, since glasso is only effective for Gaussian data. However, the marginal precision matrix $\tilde{K}_{o}$ is much denser than the conditional precision matrix $K_{o}$, and therefore, is a more complicated model (more parameters involved).

\subsection{Real Data}

The dataset consists of the expression level of 11 proteins in 7466 cells [8]. Two proteins (PKA and PCA) seem to interact with most of the 9 other proteins, and they can be considered as "hubs" (see Fig. 2(a), indicated in yellow). We remove those proteins from the dataset. The graphical models should infer those two proteins as hidden variables.

We have verified that the data is non-Gaussian, and therefore, one would expect the copula Gaussian model with hidden variables to perform well on this dataset.

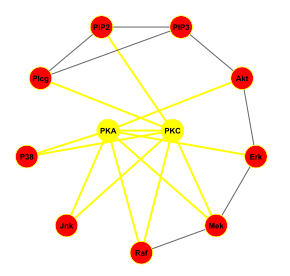

(a) True graph

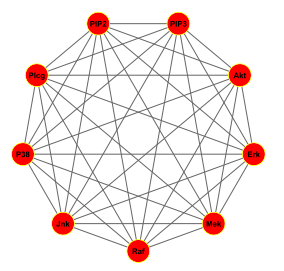

(d) GHVGM with cv

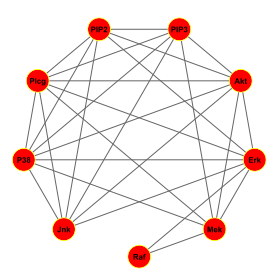

(b) Glasso

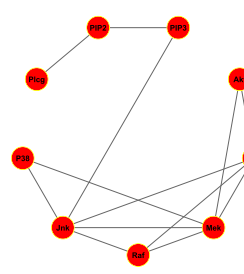

(e) CGHVGM with cv

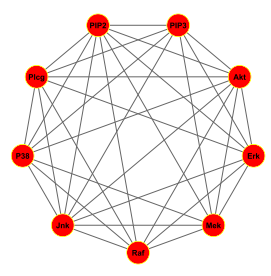

(c) Copula glasso

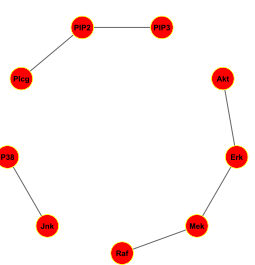

(f) CGHVGM with ss
Fig. 2. Results of different methods on the cell signaling data

The results are shown in Fig. 2. The undirected version of theoretical analysis in [8] is regarded as the "true" graph. The
CGHVGM approach with cv overfits the data. Both glasso and copula glasso are trying to estimate the marginalized dense graph. GHVGM with cv generates an incorrect full graph, and GHVGM with ss leads to a fully disconnected graph. In contrast, the proposed method yields the most accurate conditional graph. Moreover, the rank of the inferred matrix $L$ equals 2 , which is indeed the true number of hidden variables (PKA and PCA).

\section{CONCLUSION}

In this paper, we introduced the copula Gaussian graphical model with hidden variables; such model can provide a simple description of high-dimensional non-Gaussian data, where correlations can be captured through a few hidden variables. We used stability selection to learn the structure of the model and inferred the parameters of model and the number of hidden variables by solving a convex problem.

\section{REFERENCES}

[1] J. Friedman, T. Hastie, and R. Tibshirani, "Sparse inverse covariance estimation with the graphical lasso," Biostatistics 9:3, pp. 432-441, 2008.

[2] V. Chandrasekaran, P. A. Parrilo, and A. S. Willsky, "Latent Variable Graphical Model Selection via Convex Optimization," Technical report, Massachusetts Institute of Technology, 2010.

[3] A. Dobra and A. Lenkoski, "Copula Gaussian graphical models and their application to modeling functional disability data," Annals of Applied Statistics, vol. 5, No. 2A, pp. 969-993, 2011.

[4] H. Liu, K. Roeder, and L. Wasserman, "Stability Approach to Regularization Selection (StARS) for High Dimensional Graphical Models," Advances in Neural Information Processing Systems, 2010.

[5] N. Meinshausen, P. Bühlmann, "Stability Selection," Journal of the Royal Statistical Society, vol. 72, Series B, pp. 417-473, 2010.

[6] C. Wang, D. Sun, and K. C. Toh, "Solving log-determinant optimization problems by a Newton-CG primal proximal point algorithm," Society for Industrial and Applied Mathematics, vol. 20, pp. 2994-3013, 2009.

[7] H. Liu, J. Lafferty, and L. Wasserman, "The Nonparanormal: Semiparametric Estimation of High Dimensional Undirected Graphs," Journal of Machine Learning Research, pp. 22952328, 2010.

[8] K. Sachs, O. Perez, D. Peer, D. A. Lauffenburger, and G. P. Nolan "Causal Protein-Signaling Networks Derived from Multiparameter Single-Cell Data," Science Magazine vol. 308, pp. 523-529, April 2005. 